\title{
Triptolide Induces Leydig Cell Apoptosis by Disrupting Mitochondrial Dynamics in Rats
}

\author{
Linyan $\mathrm{Lv}^{1 \dagger}$, Yajie Chang ${ }^{1 \dagger}$, Yanqing $\mathrm{Li}^{1}$, Haicheng Chen ${ }^{2}$, Jiahui $\mathrm{Yao}^{2}$, Yun $\mathrm{Xie}^{2}$, \\ Xiaoyan Liang ${ }^{1}$, Xing Yang ${ }^{1 *}$, Min Zhang ${ }^{2 *}$ and Guihua Liu ${ }^{1 *}$ \\ ${ }^{1}$ Reproductive Medicine Research Center, The Sixth Affiliated Hospital of Sun Yat-sen University, Guangzhou, China, \\ ${ }^{2}$ Department of Andrology, The First Affiliated Hospital of Sun Yat-sen University, Guangzhou, China
}

OPEN ACCESS

Edited by:

Stefan Schildknecht,

University of Konstanz, Germany

Reviewed by:

Tatjana S. Kostic,

University of Novi Sad, Serbia

Yong Chen,

First Affiliated Hospital of Wenzhou

Medical University, China

${ }^{*}$ Correspondence:

Xing Yang

yxing@mail3.sysu.edu.cn

Min Zhang

zhangm287@mail.sysu.edu.cn

Guihua Liu

liuguihua@mail.sysu.edu.cn

${ }^{+}$These authors have contributed equally to this work

Specialty section:

This article was submitted to

Predictive Toxicology,

a section of the journal

Frontiers in Pharmacology

Received: 14 October 2020

Accepted: 19 January 2021

Published: 09 March 2021

Citation:

Lv L, Chang Y, Li Y, Chen H, Yao J, Xie $Y$, Liang $X$, Yang $X$, Zhang $M$ and Liu G (2021) Triptolide Induces Leydig

Cell Apoptosis by Disrupting

Mitochondrial Dynamics in Rats.

Front. Pharmacol. 12:616803.

doi: 10.3389/fphar.2021.616803
Triptolide is widely used in the clinical treatment of various diseases. Side effects, including reproductive toxicity to male patients, limit its application. However, no detailed mechanisms or potential intervention targets have been reported. In this study, we show that triptolide activated the mitochondrial apoptosis pathway in rat testicular Leydig cells and induced apoptosis both in vivo and in vitro, which may cause hypoleydigism and impair spermatogenesis. Mechanistically, triptolide-induced dynamin-related protein 1 (Drp1) overexpression, which interfered with mitochondrial dynamic stability to activate the mitochondrial apoptosis pathway. Mdivi-1, a selective Drp1 inhibitor, partially reversed the mitochondrial dynamic disturbance and rat testicular Leydig cell apoptosis induced by triptolide. Inhibiting Drp1 over-activation may be a new strategy for mitigating the reproductive toxicity of triptolide.

Keywords: triptolide, leydig cells, DRP1, mitochondrial dynamic, apoptosis

\section{INTRODUCTION}

Triptolide (TP), an active compound from a Chinese herb, is now widely used in the clinic to treat multiple diseases, including autoimmune diseases, cancers, and diabetic nephropathy. With the incidences of the diseases above increasing among males, the side effects of triptolide, especially its reproductive toxicity, limit its application (Du et al., 2018). Xiong et al. reported that triptolide decreased testicular weight, damaged testis and sperm morphology, and reduced sperm motility and density (Xiong et al., 2019). Wang et al. reported that triptolide induced oxidative stress and apoptosis in TM4 cells (Wang et al., 2018). These studies provide some clues on the reproductive toxicity of triptolide. However, more detailed mechanisms and therapeutic targets are still needed.

Testicular Leydig cells nourish the spermatogonium and provide testosterone, which is important for spermatogenesis. Mitochondria are vital for Leydig cell survival and steroidogenesis (Park et al., 2019). Triptolide causes mitochondrial membrane depolarization and activates the mitochondrial apoptosis pathway in murine pituitary corticotroph tumor cells (Li et al., 2017b). Whether triptolide damages Leydig cell mitochondria and decreases their function in spermatogenesis is still not clear.

Mitochondrial fission and fusion maintain a dynamic balance to preserve normal mitochondrial function and adapt to environmental changes (Scott and Youle, 2010). Disruption of this balance leads to mitochondrial dysfunction, which occurs in various diseases (Mouli et al., 2009; Haun et al., 2013; Khacho et al., 2014). Dynamin-related protein 1 (Drp1) is a major factor that promotes mitochondrial fission to regulate mitochondrial shape and function (Smirnova et al., 2001; Simula et al., 2019). Aberrant Drp1 expression disrupts the balance of mitochondrial fission and fusion, causing various diseases, including Parkinson's disease, pancreatic tumors, and Huntington's disease (Wang et al., 2011; Haun et al., 2013; 
Nagdas et al., 2019; Qi et al., 2019). Here, we investigated triptolideinduced aberrant Drp1 expression in Leydig cells, which disrupted the dynamic balance of mitochondrial fission and fusion, damaged mitochondrial function and finally induced steroidogenesis impairment in vivo and in vitro.

\section{MATERIALS AND METHODS}

\section{Chemical Agents}

Triptolide (TP; cat. no. HY-32735) and mitochondrial division inhibitor 1 (Mdivi-1; cat. no. HY-15886) were purchased from MedChemExpress. Briefly, $1 \mathrm{mg}$ TP was dissolved in $2.7747 \mathrm{ml}$ dimethyl sulfoxide (DMSO) to prepare stock solutions (1 mM). In vitro, the TP stock solution was diluted with culture medium to make the working solution. In vivo, TP was intragastrically administered at a dose of $400 \mu \mathrm{g} / \mathrm{kg}$ for $0-8$ weeks in different groups of rats. Ten milligrams of Mdivi-1 powder was dissolved in $2.8311 \mathrm{ml}$ DMSO to prepare stock solutions $(10 \mathrm{mM})$, and $1 \mu \mathrm{l}$ stock solution was added to $1 \mathrm{ml}$ culture medium to make the working solution $(10 \mu \mathrm{M})$.

\section{Animal Studies}

Adult male Sprague-Dawley rats (12 weeks old) were purchased from the Animal Center of Sun Yat-Sen University (Guangzhou, China). Animal studies were performed in accordance with the recommendations from the Guide for the Care and Use of Laboratory Animals of the National Institutes of Health and the Animal Welfare Act guidelines. The protocol of the present study was approved by the Ethical Committee of Sun Yat-Sen University (Guangzhou, China). Seventy-two rats were randomly divided into nine groups (eight per group) that were treated with triptolide for different periods (0-8 w). After 8 weeks, the blood and testes of rats from the different groups were collected.

\section{Histological Analysis}

Isolated rat testes were fixed with $4 \%$ paraformaldehyde for paraffin embedding and sectioned at $3 \mu \mathrm{m}$ thickness for tissue slices. Other rat testis tissues were stored in liquid nitrogen for subsequent experiments. The tissue slices were stained with hematoxylin and eosin (H\&E) using commercial kits (Servicebio, Wuhan, China) according to the manufacturer's instructions. The slices were examined by electron light microscopy (Olympus, Japan).

\section{TUNEL Assay}

Apoptosis rates in rat testis tissue were tested using a terminal deoxynucleotidyl transferase-mediated dUTP nick-end labeling (TUNEL) staining kit (Thermo Fisher Scientific, MA, United States) according to the manufacturer's instructions. After staining, the images were analyzed by confocal microscopy (Leica TCS SP8; Leica Microsystems, Inc.).

\section{Cell Culture}

The testis Leydig cell line TM3 was purchased from the Cell Bank of the Chinese Academy of Sciences (cat. no. GNM24). Cells were cultured in RPMI-1640 medium (cat. no. 12633012; Gibco; Thermo Fisher Scientific, Inc.) containing $5 \%$ fetal bovine serum (FBS; cat. no.
10099141C Gibco; Thermo Fisher Scientific, Inc.) and 2.5\% horse serum (cat. no. 26050088 Gibco; Thermo Fisher Scientific, Inc.) in a humidified incubator with $5 \% \mathrm{CO}_{2}$. After growing to $60-70 \%$ confluency, the cells were transferred to 96- or 6-well plates for different assays.

\section{Cell Apoptosis Detection}

7-Aminoactinomycin D (7-AAD) and Annexin V-allophycocyanin (APC) flow cytometry assays (cat. no. 70AP105-100; MultiSciences) were used to detect cell apoptosis according to the manufacturer's instructions. Briefly, cells were harvested after different treatments. After washing with cold PBS buffer three times, the cell pellets were resuspended in binding buffer and stained with APC-conjugated Annexin V and 7-AAD for $10 \mathrm{~min}$. Then, the cells were analyzed by flow cytometry (FACS Canto II; Becton, Dickinson and Company).

\section{Cell Viability Detection}

A total of 2,000 cells per well were seeded into 96-well plates. After culturing for $12 \mathrm{~h}$, different treatments were added according to the corresponding experimental conditions. Then, $10 \mu \mathrm{l}$ of Cell Counting Kit-8 reagent (CCK-8; cat. no. CK04; Dojindo Molecular Technologies, Inc.) was added to each well and incubated for an additional $1.5 \mathrm{~h}$. The optical density values were measured at a $450-\mathrm{nm}$ wavelength on a microplate reader (Multiskan $^{\mathrm{TM}}$ FC; Thermo Fisher Scientific, Inc.).

\section{ROS Assay}

CellROX Oxidative Stress Reagents (cat. no. C10443; Thermo Fisher Scientific, Inc.) were used to detect cell ROS according to the manufacturer's instructions. The cells were incubated with serumfree medium containing $5 \mu \mathrm{M}$ CellROX Reagent for $30 \mathrm{~min}$ at $37^{\circ} \mathrm{C}$ after different treatments and then washed with PBS three times. The cells were observed under a laser scanning confocal microscope (Leica TCS SP8; Leica Microsystems, Inc.).

\section{Mitochondrial Membrane Potential (MMP) Assay}

Tetramethylrhodamine (TMRM; cat. no. I34361; Thermo Fisher Scientific, Inc.) was used to detect MMP according to the manufacturer's instructions. Briefly, cells in different groups were stained with $50 \mathrm{nmol} / \mathrm{l}$ TMRM in serum-free medium at $37^{\circ} \mathrm{C}$ for $30 \mathrm{~min}$, washed with PBS three times, and then observed under a laser scanning confocal microscope (Leica TCS SP8; Leica Microsystems, Inc.) or analyzed by flow cytometry (FACS Canto II; Becton, Dickinson and Company).

\section{Western Blot Analysis}

TM3 cells ( $10^{6}$ cells) or $20 \mathrm{mg}$ testicular tissue was added to $100 \mu \mathrm{l}$ radioimmunoprecipitation assay lysis buffer (cat. no. P0013B; Beyotime Institute of Biotechnology) with phenylmethanesulfonyl fluoride (cat. no. ST506; Beyotime Institute of Biotechnology). The concentrations of cell lysates were quantified by a Pierce BCA Protein Assay Kit (cat. no. 23227; Thermo Fisher Scientific, Inc.). Antibodies against Drp1, Mfn1, Fis-1, OPA1, and Bcl-2 were purchased from Abcam (cat. nos. ab184247, ab106274, ab157457, and ab59348). 

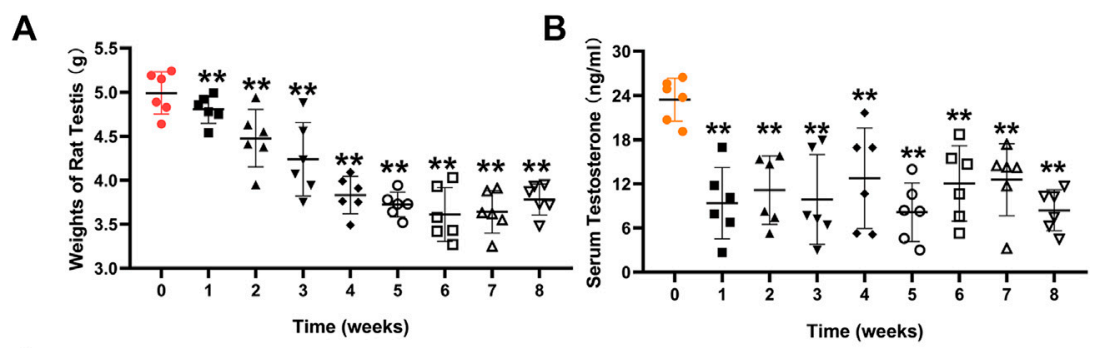

C
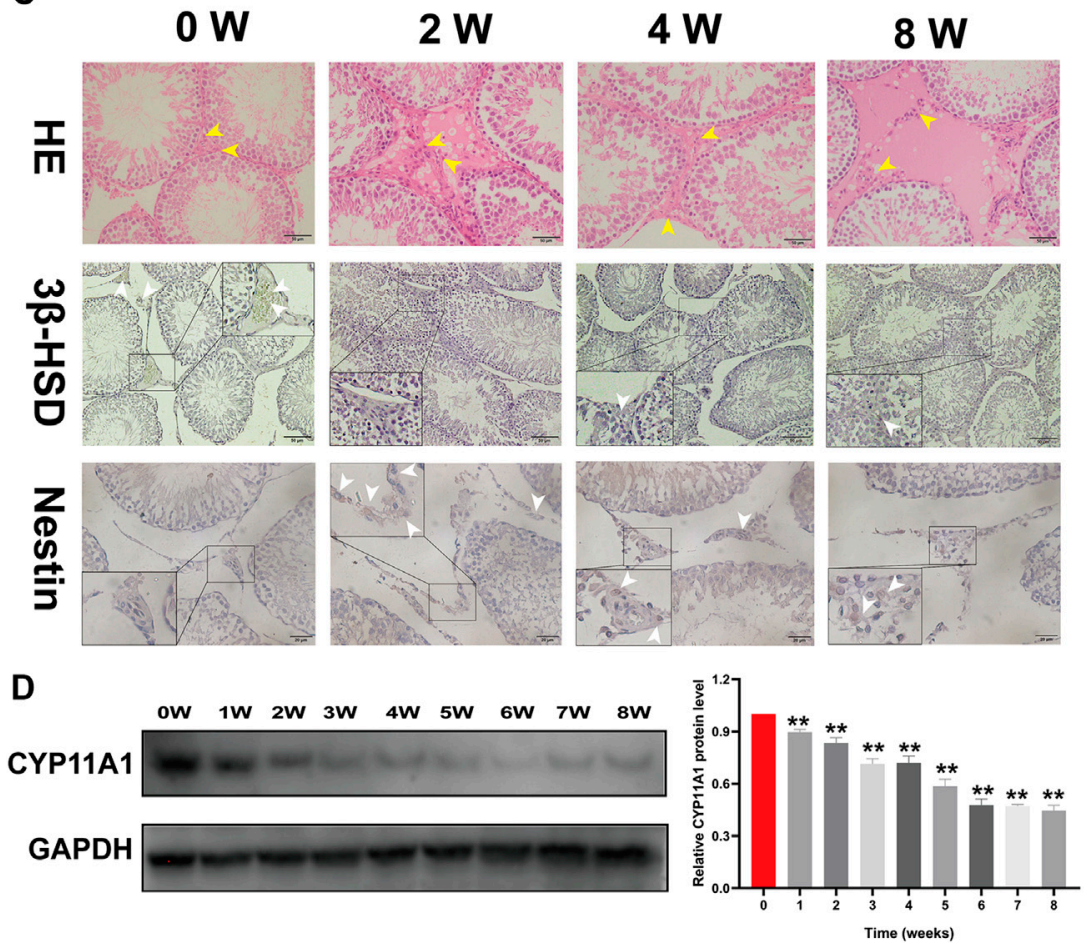

FIGURE 1 | Triptolide damages the interstitial tissue of rat testes and decreases serum testosterone levels in rats. (A) Weights of rat testes in each group $(n=6 /$ group) were measured. (B) Serum testosterone levels of rats in each group ( $n=6$ /group) were detected using an ELISA kit. (C) First line: Representative images of HEstained rat testes. Second line: Representative images of 3 $\beta-H S D$ immunostaining in rat testes. Third line: Representative images of nestin immunostaining in rat testes. (D) Western blot analysis of CYP11A1 protein levels in rat testes. Data represent the mean \pm SD $(n=6 /$ group) for panels (A,B). Data represent the mean \pm SD from three independent experiments for panels (C,D). Group comparisons were performed by one-way analysis of variance followed by Tukey's post hoc test. ${ }^{\star \star} p<0.01$ vs. the 0 -week group. ${ }^{\star} p<0.05$ vs. the 0 -week group. White arrows indicate positively stained cells in the IHC assay. Yellow arrows indicate cells in the interstitial tissue of rat testes.

Antibodies against Bax, cleaved caspase 3, and caspase 3 were purchased from Cell Signaling Technology (cat. nos. 5023, 9661, and 9662, respectively). Antibodies against GAPDH were purchased from ProteinTech Group, Inc. (cat. no. 10494-1-AP). HRPconjugated secondary antibody was obtained from CWBIO (cat. no. CW0103).

\section{Blood Sample Collection and ELISA}

Blood samples were collected from the abdominal aorta after triptolide or placebo administration. After incubation at room temperature for $1 \mathrm{~h}$, the blood samples were centrifuged at $1,500 \mathrm{rpm}$ for $20 \mathrm{~min}$, and the upper serum was collected. Serum testosterone levels of rats were detected by using a Rat Testosterone ELISA Kit (cat. no. CSB-E05100r; Cusabio) according to the manufacturer's instructions.

\section{Immunohistochemistry}

The protein level of Drp1 in testis tissue was detected as described before. Briefly, testis tissue slices from different rats were incubated with anti-Drp1 (1:150, ab184247, Abcam) antibody overnight at $4^{\circ} \mathrm{C}$ followed by incubation with a corresponding horseradish peroxidase-conjugated secondary antibody and hematoxylin staining. Randomly selected sections of the tissue slices were examined using light microscopy (Olympus, Japan).

\section{Statistical Analysis}

Data represent the mean \pm SD from three independent experiments and were analyzed using SPSS 25.0 (IBM Corp.). One-way analysis of variance followed by Tukey's post hoc test was used to measure differences between groups. A difference 


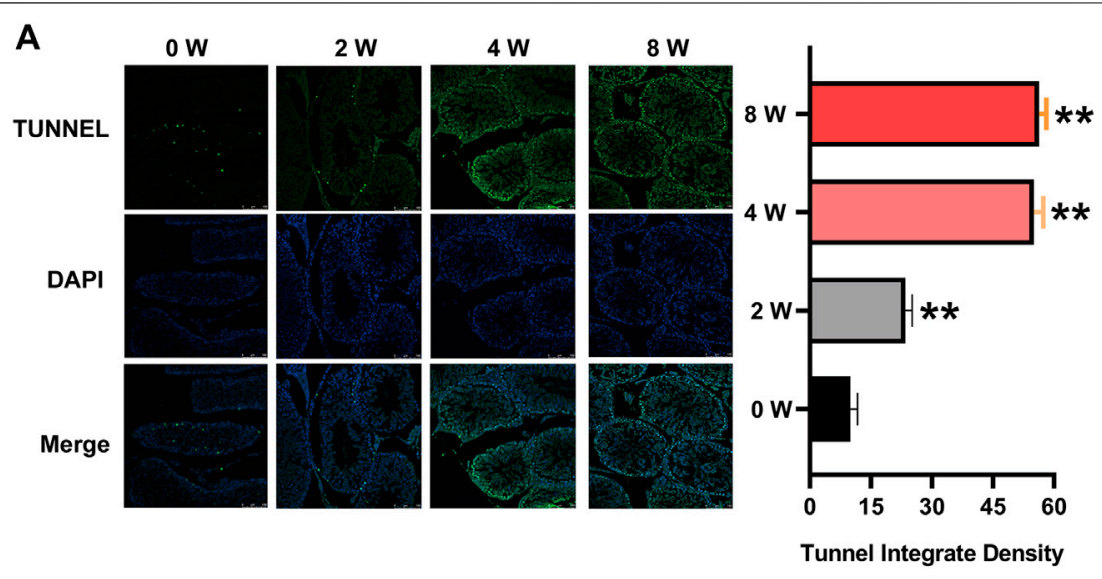

B

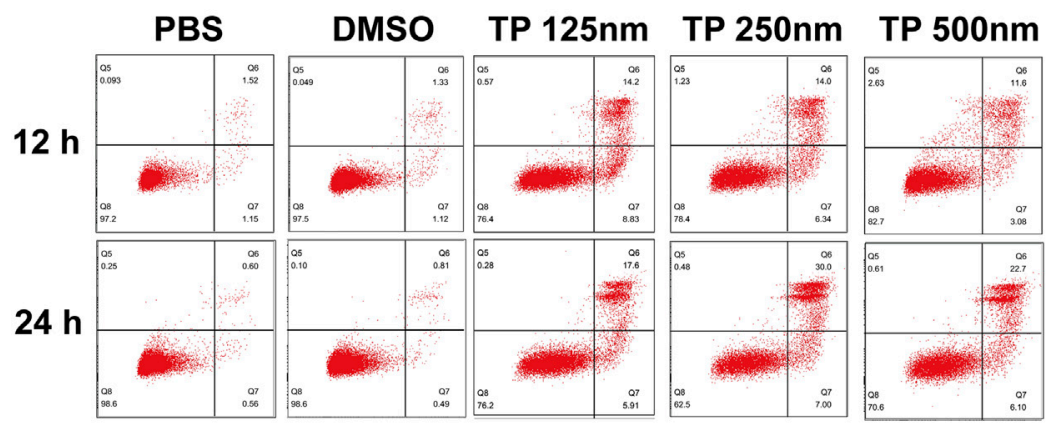

C

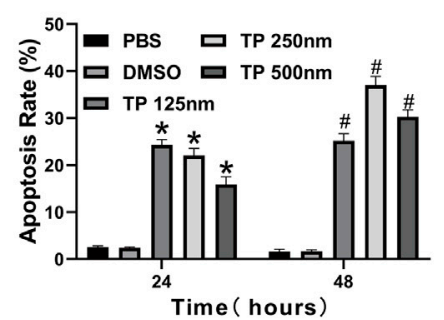

D

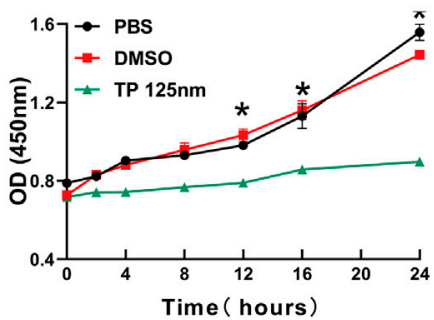

FIGURE 2 | Triptolide induces Leydig cell apoptosis in vitro and in vivo. (A) Representative TUNEL staining images of rat testes. (B) Annexin V-FITC/PI apoptosis assays of TM3 cells. (C) Quantitative analysis of the results shown in (B). (D) CCK-8 assay of TM3 cells. Data represent the mean \pm SD from three independent experiments. Group comparisons were performed by one-way analysis of variance followed by Tukey's post hoc test. ${ }^{\star \star}<0.01$ vs. the DMSO group at 24 h, ${ }^{\star} p<0.05$ vs. the DMSO group at $24 \mathrm{~h}{ }^{*} p<0.05$ vs. the DMSO group at $48 \mathrm{~h}$.

with a $p$ value less than 0.05 was considered statistically significant.

\section{RESULT}

1. Triptolide damages rat testes and decreases testosterone synthesis-associated enzyme levels.

- To identify the effect of triptolide on rat testes, we measured testicular weights, diameters, and serum testosterone levels of rats in different groups. We found that the weights and testosterone levels were both reduced after triptolide treatment (Figure 1A,B). However, no significant difference was observed in diameter (SupplementaryFigure S1). Then, we conducted HE staining of rat testes. The results showed obvious structural disorder, including decreased cell numbers and vacuolation in the interstitial tissue of rat testes after 2 weeks of triptolide treatment (Figure 1C), which showed that triptolide damaged the interstitial tissue of rat testes. The protein levels of $3 \beta-H S D$ and CYP11A1 (testosterone synthesis-associated enzymes) were significantly decreased after triptolide treatment (Figure 1C). However, the expression of nestin, an important marker of Leydig stem cells whose expression is increased after injury (Guo et al., 2013), was increased after triptolide treatment (Figure 1C). All these results demonstrate that triptolide damaged the interstitial tissue of rat testes, which might not be detectable by measuring testicular diameter or volumes. 


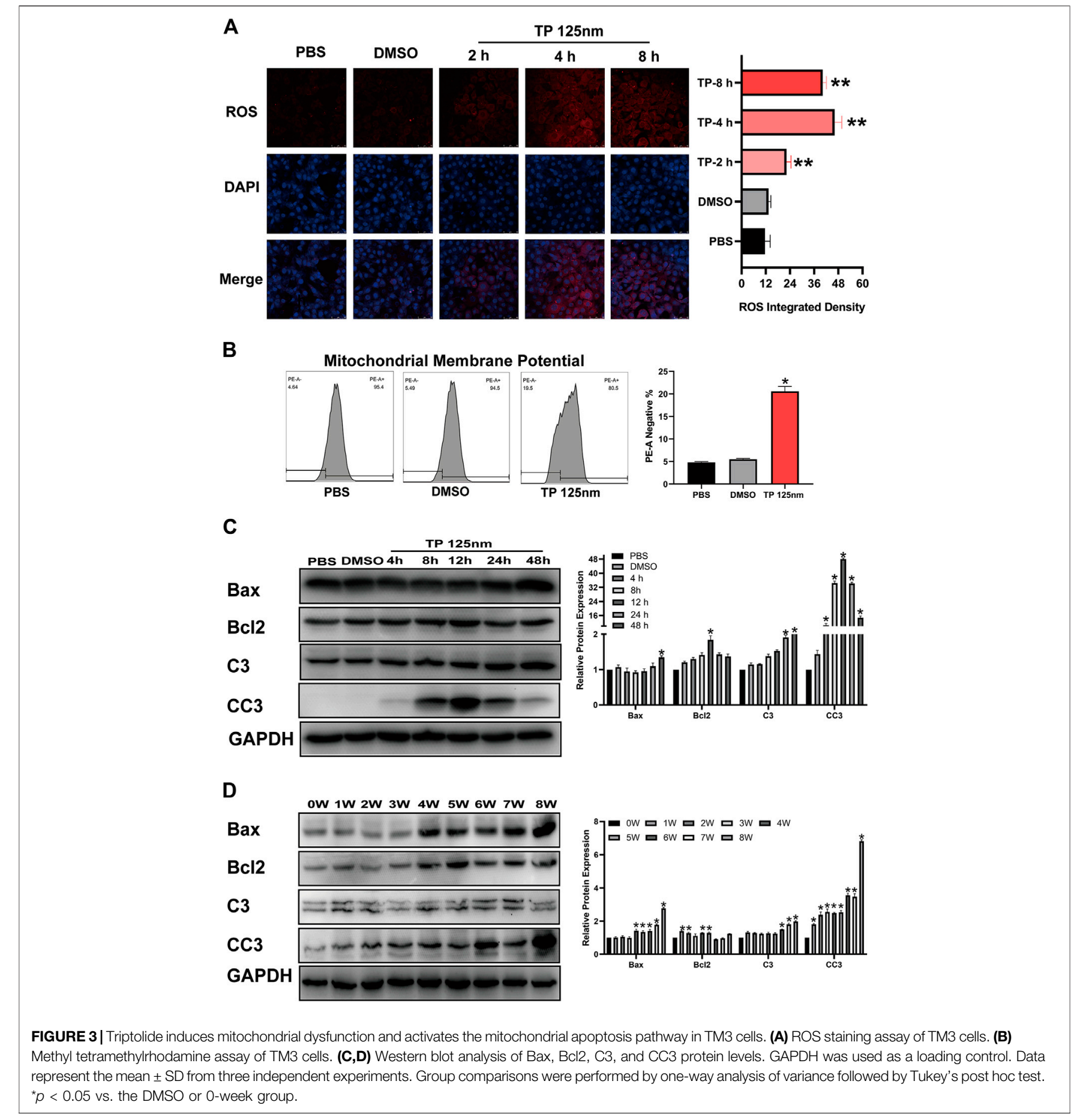

\section{Triptolide Induces Rat Leydig Cell Apoptosis and Inhibits Leydig Cell Viability In Vitro and In Vivo}

After we found that triptolide damaged the interstitial tissue of rat testes, we next investigated the cause of this damage. TUNEL staining of testis tissue showed a time-dependent increase in apoptotic cells in the interstitial tissue of the testis after triptolide treatment. One of the important cell types in the interstitial tissue of the testis is the Leydig cell, so we chose the Leydig cell line TM3 to determine the effect of triptolide in vitro. The results showed that triptolide induced apoptosis of TM3 cells (Figure 2B). Moreover, the CCK-8 assay showed that triptolide significantly decreased the viability of TM3 cells (Figure 2D).

3. Triptolide damages mitochondrial function and activates the mitochondrial apoptosis pathway in vitro and in vivo. 


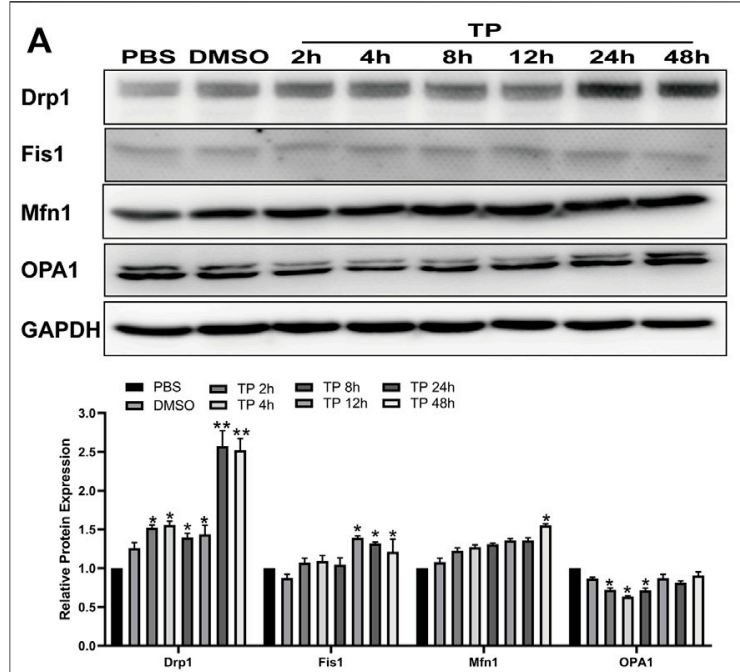

B
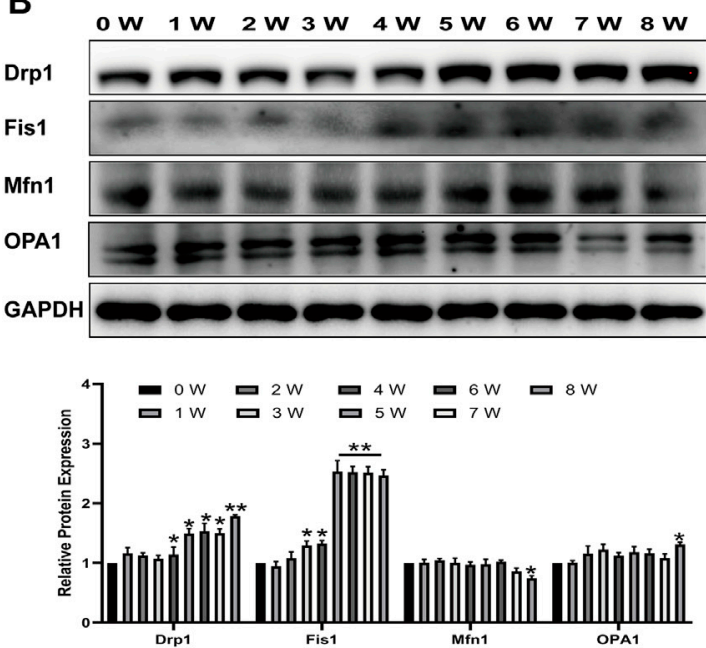

C

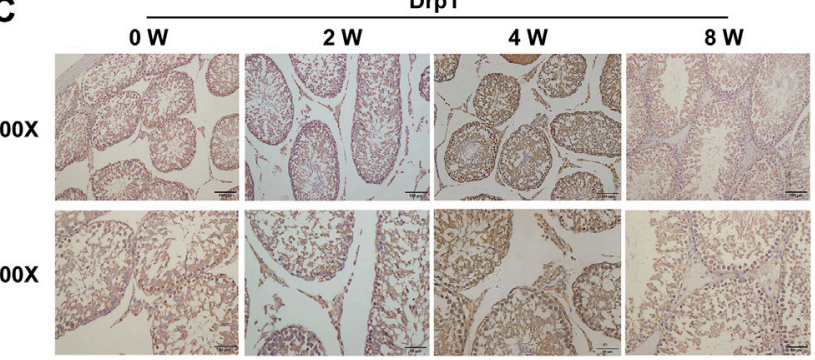

FIGURE 4 | Triptolide increases Drp1 expression and disrupts mitochondrial dynamics in vitro and in vivo. (A,B) Western blot analysis of Drp1, Mfn1, OPA1, and Fis1 protein levels. GAPDH was used as a loading control. (C) Representative images of Drp1 immunostaining in rat testes. Data represent the mean \pm SD from three independent experiments. Group comparisons were performed by one-way analysis of variance followed by Tukey's post hoc test. ${ }^{* \star} p<0.01$ vs. the DMSO or 0 -week group. ${ }^{*} p<0.05$ vs. the DMSO or 0-week group.

Normal mitochondrial function is very important for Leydig cell survival, so we determined whether triptolide induces cell apoptosis by inducing mitochondrial dysfunction. The results showed that triptolide increased ROS levels (Figure 3A) and reduced mitochondrial membrane potential after triptolide treatment in TM3 cells (Figure 3B). Severe mitochondrial injury activates the mitochondrial apoptosis pathway and induces cell apoptosis, so we determined whether the mitochondrial apoptosis pathway is activated after triptolide

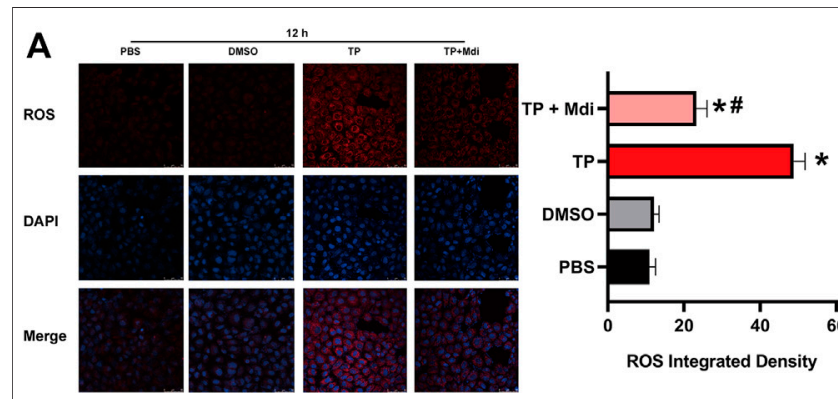

B

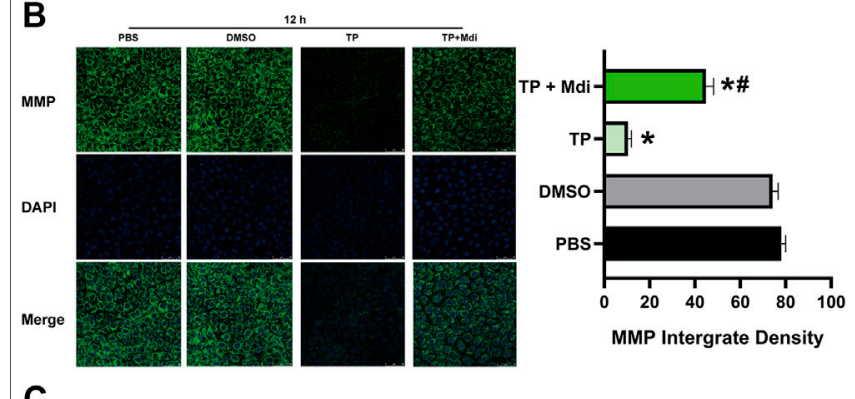

C

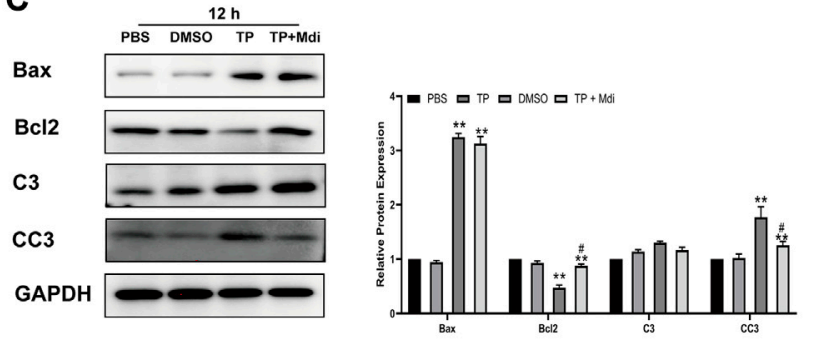

D

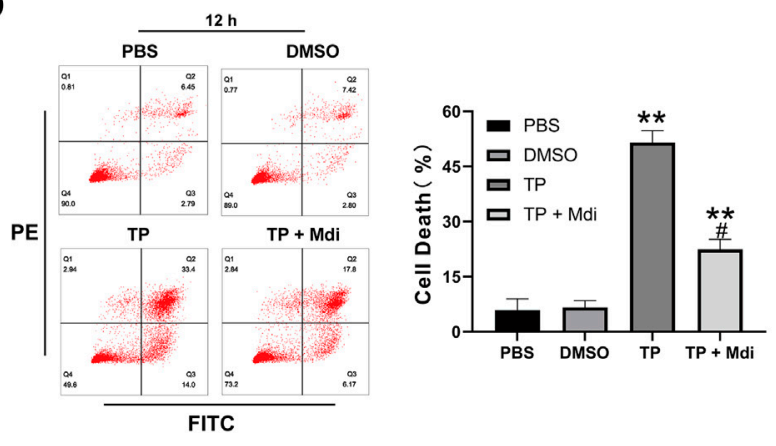

FIGURE 5 | Mdivi-1 partially reverses triptolide-induced mitochondrial dynamic disruption, mitochondrial dysfunction and cell apoptosis in vitro. (A) ROS staining assay of TM3 cells. (B) Methyl tetramethylrhodamine assay of TM3 cells. (C) Western blot analysis of Bax, Bcl2, C3, and CC3 protein levels. GAPDH was used as a loading control. (D) Annexin V-FITC/PI apoptosis assays of TM3 cells. Data represent the mean \pm SD from three independent experiments. Group comparisons were performed by one-way analysis of variance followed by Tukey's post hoc test. ${ }^{* *} p<0.01$ vs. the DMSO or 0 -week group. ${ }^{*} p<0.05$ vs. the DMSO or 0 -week group. 
treatment. The results showed that the expression levels of key factors in the mitochondrial apoptosis pathway, including Bax and CC3, were significantly increased in vitro and in vivo (Figures 3C,D), which indicates that mitochondrial apoptosis pathway activation may be the key step in triptolide-induced cell apoptosis.

\section{Triptolide Disrupts the Dynamic Mitochondrial Balance in Leydig Cells and Rat Testis}

Next, we studied how triptolide causes mitochondrial dysfunction. Mitochondria are in a highly balanced state of fission and fusion, and disruption of this balance leads to mitochondrial dysfunction. Here, we found that triptolide significantly increased the expression of dynamin-related protein 1 (Drp1), a key factor in promoting mitochondrial fission, in vitro and in vivo, ultimately disrupting the dynamic balance of mitochondrial fission and fusion (Figures $4 \mathbf{A}, \mathbf{B}$ ). Immunohistochemical analysis of rat testis slices showed that the expression of Drp1 was increased in the interstitial tissue of rat testes (Figure 4C).

5. Mdivi-1 partially reverses the disruption of mitochondrial dynamic balance, mitochondrial dysfunction, and activation of the mitochondrial apoptosis pathway induced by triptolide.

Triptolide causes mitochondrial dysfunction by disrupting the dynamic balance of mitochondrial fission and fusion, which activates the mitochondrial apoptosis pathway and finally induces cell apoptosis. We hypothesized that reversing the excessive mitochondrial fission induced by triptolide could preserve the normal mitochondrial function of cells and ultimately reduce cell apoptosis. We used Mdivi-1, a specific Drp1 inhibitor, to inhibit mitochondrial fission induced by triptolide. The results showed that Mdivi-1 reduced the expression of Drp1 (Figure 5A) and restored the dynamic balance of mitochondrial fission and fusion. Moreover, Mdivi1 decreased the ROS level (Figure 5B), increased the mitochondrial membrane potential (Figure 5C), and inhibited mitochondrial apoptosis pathway activation after triptolide treatment (Figure 5D).

\section{DISCUSSION}

In the present study, we found that triptolide reduced testicular weight and serum testosterone in rats and induced Leydig cell apoptosis, mitochondrial dysfunction and interstitial tissue damage by disrupting the dynamic balance of mitochondrial fission and fusion. This effect was partially reversed by the dynamin-related protein-1 inhibitor Mdivi-1.

Triptolide is widely used in the clinic for its biological effects, which include anti-inflammatory, anti-rheumatoid, and antitumor effects (Liu, 2011). However, the reproductive toxicity of triptolide substantially limits its application in male patients with fertility needs. Triptolide was shown to cause infertility and abnormal spermatogenesis in male rats (Huynh et al., 2000; Huang et al., 2015). This effect was thought to be caused by triptolide directly damaging spermatogonia, but whether other cell types, including Leydig cells, participate in this process is not clear. Previous studies have shown that Leydig cells play an important role in testosterone production and spermatogenesis (Zirkin and Papadopoulos, 2018), and triptolide was shown to inhibit superoxide dismutase expression, which increased intracellular ROS in rat Leydig cells, finally inducing oxidative stress damage and cell death (Hu et al., 2015). In addition, triptolide increased miR-26a expression in rat Leydig cells, which decreased GSk $3 \beta$ mRNA levels and inhibited testosterone production (Liang et al., 2019). Another study also reported that triptolide induced Leydig cell apoptosis, and this effect might be highly associated with miR-200 (Miao et al., 2020). These results suggested that miRNAs might play an important role in triptolide-induced Leydig cell apoptosis. In our study, we found that triptolide reduced testicular weight and serum testosterone levels but did not influence testicular volume. HE staining showed interstitial vacuolation and a significantly decreased cell number both inside and outside of the seminiferous tubule. Furthermore, we found that triptolide-induced Leydig cell death in vivo and in vitro, which might contribute to the decrease in serum testosterone. More interestingly, we observed that the protein levels of nestin, which is a marker of stem Leydig cells, increased after triptolide treatment. A previous study reported that stem Leydig cells could proliferate and differentiate into mature Leydig cells after Leydig cell loss (Guo et al., 2013; Chen et al., 2017). However, it is unclear whether triptolide promotes stem Leydig cell proliferation through other mechanisms; this possibility needs further exploration.

Mitochondria are very important for Leydig cell survival and testosterone production (Kong et al., 2017a). Previous studies have revealed that mitochondrial dysfunction of Leydig cells significantly reduced testosterone production and serum testosterone ( $\mathrm{Li}$ et al., 2017a; Kong et al., 2017b; Goncalves et al., 2018). Here, we found that triptolide activated the mitochondrial apoptosis pathway, increased cell ROS, and depolarized mitochondrial membrane potential. According to these data, we conclude that triptolide damaged Leydig cell mitochondria and inhibited testosterone production, which might play a significant role in triptolide-induced sperm abnormalities.

The balanced state of mitochondrial dynamics, including mitochondrial fission and fusion, is crucial for normal mitochondrial function (Whitley et al., 2019). Research has shown that triptolide induces excessive activation of mitophagy, which leads to mitochondrial dysfunction and cell death (Hasnat et al., 2019). In addition, ageing-related mitochondrial dynamic disruption was associated with decreased serum testosterone (Sokanovic et al., 2020). Here, we found that triptolide significantly increased the expression of mitochondrial fission proteins, including dynamin-related protein 1 (drp1) and FIS1. Moreover, the expression of mitochondrial fusion proteins, including mitofusion 1 and optic atrophy type 1 (OPA1), was decreased after triptolide treatment. The changes in these proteins indicated that triptolide promoted mitochondrial fission 
and disrupted the balance of mitochondrial dynamics (Meyer et al., 2017; Tilokani et al., 2018). Because drp1 is the key factor in mitochondrial fission (Chandra et al., 2017; Ding et al., 2018), we explored whether inhibiting drp1 could alleviate triptolide-induced cell damage. Mitochondrial division inhibitor 1 (Mdivi-1) is a highly efficient Drp1 inhibitor that can mitigate excessive Drp1-dependent mitochondrial fission and subsequent cell apoptosis in different diseases (Fan et al., 2017; Ruiz et al., 2018; Wu et al., 2018). In our study, we found that Mdivi-1 reversed triptolide-induced mitochondrial dynamic instability to mitigate mitochondrial dysfunction, ultimately inhibiting cell apoptosis. These results suggest that triptolide disrupts the balance of mitochondrial dynamics in Leydig cells in a Drp1-dependent manner. Restoring the balance of mitochondrial dynamics may be an effective method to mitigate the reproductive toxicity of triptolide, and Drp1 is an ideal therapeutic target for this purpose. Highly efficient and specific inhibitors of Drp1 are needed.

\section{DATA AVAILABILITY STATEMENT}

The original contributions presented in the study are included in the article/Supplementary Material, further inquiries can be directed to the corresponding authors.

\section{ETHICS STATEMENT}

The animal study was reviewed and approved by the Institutional Animal Care and Use Committee (IACUC), Sun Yat-Sen University.

\section{REFERENCES}

Chandra, R., Engeln, M., Schiefer, C., Patton, M. H., Martin, J. A., Werner, C. T., et al. (2017). Drp1 mitochondrial fission in D1 neurons mediates behavioral and cellular plasticity during early cocaine abstinence. Neuron 96 (6), 1327-1341. doi:10.1016/j.neuron.2017.11.037

Chen, H., Wang, Y., Ge, R., and Zirkin, B. R. (2017). Leydig cell stem cells: identification, proliferation and differentiation. Mol. Cell Endocrinol. 445, 65-73. doi:10.1016/j.mce.2016.10.010

Ding, M., Feng, N., Tang, D., Feng, J., Li, Z., Jia, M., et al. (2018). Melatonin prevents Drp1-mediated mitochondrial fission in diabetic hearts through SIRT1-PGC1a pathway. J. Pineal. Res. 65 (2), e12491. doi:10.1111/jpi. 12491

Du, X., Nyagblordzro, M., An, L., Gao, X., Du, L., Wang, Y., et al. (2018). Pharmacokinetic and toxicological characteristics of tripterigium glycosides and their derivatives. Curr. Drug Metab. 19 (7), 605-627. doi:10.2174/ 1389200219666180302152752

Fan, L. F., He, P. Y., Peng, Y. C., Du, Q. H., Ma, Y. J., Jin, J. X., et al. (2017). Mdivi-1 ameliorates early brain injury after subarachnoid hemorrhage via the suppression of inflammation-related blood-brain barrier disruption and endoplasmic reticulum stress-based apoptosis. Free Radic. Biol. Med. 112, 336-349. doi:10.1016/j.freeradbiomed.2017.08.003

Guo, J., Li, X., Liang, Y., Ge, Y., Chen, X., Lian, Q., et al. (2013). The increased number of Leydig cells by di(2-ethylhexyl) phthalate comes from the differentiation of stem cells into Leydig cell lineage in the adult rat testis. Toxicology 306, 9-15. doi:10.1016/j.tox.2013.01.021

Goncalves, G. D., Semprebon, S. C., Biazi, B. I., Mantovani, M. S., and Fernandes, G. (2018). Bisphenol A reduces testosterone production in TM3 Leydig cells

\section{AUTHOR CONTRIBUTIONS}

GL, XY, and MZ contributed to the design of the study. LL, YC, YL, HC, YX, and JY performed the experiments. GL, XL, and LL performed the statistical analyses. LYL wrote the first draft of the manuscript. YC, XY, and YL wrote sections of the manuscript. All authors contributed to manuscript revision and read and approved the submitted version.

\section{FUNDING}

This work was supported by the National Natural Science Foundation of China $(81401197,81671834,81873829$, and 81971759); the Natural Science Foundation of Guangdong Province, China (2016A030313229, 2017A030313784, and 2018A030310286); the Health Care Collaborative Innovation Foundation Major Projects of Guangzhou City, Guangdong Province, China (201604020189); the Guangdong Special Support Plan-Science and Technology Innovation Youth Top Talents Project (2016TQ03R444); the Pearl River S\&T Nova Program of Guangzhou (201806010089); and the Youth Teacher Training Project of Sun Yat-sen University (17ykpy68 and 18ykpy09).

\section{SUPPLEMENTARY MATERIAL}

The Supplementary Material for this article can be found online at: https:/www.frontiersin.org/articles/10.3389/fphar.2021.616803/ full\#supplementary-material.

independently of its effects on cell death and mitochondrial membrane potential. Reprod. Toxicol. 76, 26-34. doi:10.1016/j.reprotox.2017.12.002

Hasnat, M., Yuan, Z., Naveed, M., Khan, A., Raza, F., Xu, D., et al. (2019). Drp1associated mitochondrial dysfunction and mitochondrial autophagy: a novel mechanism in triptolide-induced hepatotoxicity. Cell Biol. Toxicol. 35 (3), 267-280. doi:10.1007/s10565-018-9447-8

Haun, F., Nakamura, T., Shiu, A. D., Cho, D., Tsunemi, T., Holland, E. A., et al. (2013). S-nitrosylation of dynamin-related protein 1 mediates mutant huntingtin-induced mitochondrial fragmentation and neuronal injury in huntington's disease. Antioxid. Redox Signal 19 (11), 1173-1184. doi:10. 1089/ars.2012.4928

Hu, J., Yu, Q., Zhao, F., Ji, J., Jiang, Z., Chen, X., et al. (2015). Protection of Quercetin against Triptolide-induced apoptosis by suppressing oxidative stress in rat Leydig cells. Chem. Biol. Interact. 240, 38-46. doi:10.1016/j.cbi.2015. 08.004

Huynh, P. N., Hikim, A. P., Wang, C., Stefonovic, K., Lue, Y. H., Leung, A., et al. (2000). Long-term effects of triptolide on spermatogenesis, epididymal sperm function, and fertility in male rats. J. Androl. 21 (5), 689-699. doi:10.1002/j. 1939-4640.2000.tb02137.x

Huang, Z. J., Que, H. Q., Peng, H. Y., Lin, S., Guo, S. M., and Qian, L. P. (2015). [Reproductive toxicity of triptolide and its mechanism in male rats]. Zhongguo Zhong Yao Za Zhi 40 (23), 4655-4659. doi:10.4268/cjcmm20152322

Khacho, M., Tarabay, M., Patten, D., Khacho, P., MacLaurin, J. G., Guadagno, J., et al. (2014). Acidosis overrides oxygen deprivation to maintain mitochondrial function and cell survival. Nat. Commun. 5 (1), 3550. doi:10.1038/ncomms4550

Kong, D., Zhang, J., Hou, X., Zhang, S., Tan, J., Chen, Y., et al. (2017b). Acetamiprid inhibits testosterone synthesis by affecting the mitochondrial function and cytoplasmic adenosine triphosphate production in rat Leydig cells. Biol. Reprod. 96 (1), 254-265. doi:10.1095/biolreprod.116.139550 
Kong, D., Zhang, J., Hou, X., Zhang, S., Tan, J., Chen, Y., et al. (2017a). Acetamiprid inhibits testosterone synthesis by affecting the mitochondrial function and cytoplasmic adenosine triphosphate production in rat Leydig cellsdagger. Biol. Reprod. 96 (4), 254-265. doi:10.1095/biolreprod.116.139550

Li, C. J., Jiang, Y. W., Chen, S. X., Li, H. J., Chen, L., Liu, Y. T., et al. (2017a). 4Methylcatechol inhibits cell growth and testosterone production in TM3 Leydig cells by reducing mitochondrial activity. Andrologia 49 (1), 1-6. doi:10.1111/ and.12581

Li, R., Zhang, Z., Wang, J., Huang, Y., Sun, W., Xie, R., et al. (2017b). Triptolide suppresses growth and hormone secretion in murine pituitary corticotroph tumor cells via NF-kappaB signaling pathway. Biomed. Pharmacother. 95, 771-779. doi:10.1016/j.biopha.2017.08.127

Liang, H., Zhang, S., and Li, Z. (2019). Ginsenoside Rg3 protects mouse leydig cells against triptolide by downregulation of miR-26a. Drug Des. Devel. Ther. 13, 2057-2066. doi:10.2147/DDDT.S208328

Liu, Q. (2011). Triptolide and its expanding multiple pharmacological functions. Int. Immunopharmacol. 11 (3), 377-383. doi:10.1016/j.intimp.2011.01.012

Meyer, J. N., Leuthner, T. C., and Luz, A. L. (2017). Mitochondrial fusion, fission, and mitochondrial toxicity. Toxicology 391, 42-53. doi:10.1016/j.tox.2017. 07.019

Miao, H., Miao, C., Han, J., and Li, N. (2020). Downregulation of miR-200a protects mouse leydig cells against triptolide by triggering autophagy. Drug Des. Devel. Ther. 14, 4845-4854. doi:10.2147/DDDT.S269236

Mouli, P. K., Twig, G., and Shirihai, O. S. (2009). Frequency and selectivity of mitochondrial fusion are key to its quality maintenance function. Biophys. J. 96 (9), 3509-3518. doi:10.1016/j.bpj.2008.12.3959

Nagdas, S., Kashatus, J. A., Nascimento, A., Hussain, S. S., Trainor, R. E., Pollock, S. R., et al. (2019). Drp1 promotes KRas-driven metabolic changes to drive pancreatic tumor growth. Cell Rep. 28 (7), 1845-1859. doi:10.1016/j.celrep. 2019.07.031

Park, J. E., Kim, Y. J., Lee, S. G., Kim, J. Y., Chung, J. Y., Jeong, S. Y., et al. (2019). Drp1 phosphorylation is indispensable for steroidogenesis in leydig cells. Endocrinology 160 (4), 729-743. doi:10.1210/en.2019-00029

Qi, Z., Huang, Z., Xie, F., and Chen, L. (2019). Dynamin-related protein 1: a critical protein in the pathogenesis of neural system dysfunctions and neurodegenerative diseases. J. Cell Physiol. 234 (7), 10032-10046. doi:10. 1002/jcp. 27866

Ruiz, A., Alberdi, E., and Matute, C. (2018). Mitochondrial division inhibitor 1 (mdivi-1) protects neurons against excitotoxicity through the modulation of mitochondrial function and intracellular Ca2+ signaling. Front. Mol. Neurosci. 11, 3. doi:10.3389/fnmol.2018.00003

Scott, I., and Youle, R. J. (2010). Mitochondrial fission and fusion. Essays Biochem. 47, 85-98. doi:10.1042/bse0470085
Simula, L., Campanella, M., and Campello, S. (2019). Targeting Drp1 and mitochondrial fission for therapeutic immune modulation. Pharmacol. Res. 146, 104317. doi:10.1016/j.phrs.2019.104317

Smirnova, E., Griparic, L., Shurland, D. L., and van der Bliek, A. M. (2001). Dynamin-related protein Drp1 is required for mitochondrial division in mammalian cells. Mol. Biol. Cell 12 (8), 2245-2256. doi:10.1091/mbc.12.8.2245

Sokanovic, S. J., Baburski, A. Z., Kojic, Z., Medar, M. L. J., Andric, S. A., and Kostic, T. S (2020). Aging-related increase of cGMP disrupts mitochondrial homeostasis in leydig cells. J. Gerontol. Ser. A 76, 177. doi:10.1093/gerona/glaa132

Tilokani, L., Nagashima, S., Paupe, V., and Prudent, J. (2018). Mitochondrial dynamics: overview of molecular mechanisms. Essays Biochem. 62 (3), 341-360. doi:10.1042/EBC20170104

Wang, H., Chen, L., and Ye, X. Y. (2018). [Triptolide induces oxidative stress and apoptosis and activates PIK3/Akt signaling pathway in TM4 sertoli cells]. Beijing Da Xue Xue Bao Yi Xue Ban 50 (4), 607-612.

Wang, H., Song, P., Du, L., Tian, W., Yue, W., Liu, M., et al. (2011). Parkin ubiquitinates Drp1 for proteasome-dependent degradation: implication of dysregulated mitochondrial dynamics in Parkinson disease. J. Biol. Chem. 286 (13), 11649-11658. doi:10.1074/jbc.M110.144238

Whitley, B. N., Engelhart, E. A., and Hoppins, S. (2019). Mitochondrial dynamics and their potential as a therapeutic target. Mitochondrion 49, 269-283. doi:10. 1016/j.mito.2019.06.002

Wu, Q., Gao, C., Wang, H., Zhang, X., Li, Q., Gu, Z., et al. (2018). Mdivi-1 alleviates blood-brain barrier disruption and cell death in experimental traumatic brain injury by mitigating autophagy dysfunction and mitophagy activation. Int. J. Biochem. Cell Biol. 94, 44-55. doi:10.1016/j.biocel.2017.11.007

Xiong, S., Li, Y., Xiang, Y., Peng, N., Shen, C., Cai, Y., et al. (2019). Dysregulation of IncRNA and circRNA expression in mouse testes after exposure to triptolide. Curr. Drug. Metab. 20 (8), 665-673. doi:10.2174/1389200220666190729130020

Zirkin, B. R., and Papadopoulos, V. (2018). Leydig cells: formation, function, and regulation. Biol. Reprod. 99 (1), 101-111. doi:10.1093/biolre/ioy059

Conflict of Interest: The authors declare that the research was conducted in the absence of any commercial or financial relationships that could be construed as a potential conflict of interest.

Copyright (c) 2021 Lv, Chang, Li, Chen, Yao, Xie, Liang, Yang, Zhang and Liu. This is an open-access article distributed under the terms of the Creative Commons Attribution License (CC BY). The use, distribution or reproduction in other forums is permitted, provided the original author(s) and the copyright owner(s) are credited and that the original publication in this journal is cited, in accordance with accepted academic practice. No use, distribution or reproduction is permitted which does not comply with these terms. 